\title{
Medical management of ectopic pregnancy - A case History
}

\author{
S Akhter ${ }^{1}$, R Nazneen ${ }^{2}$
}

\begin{abstract}
Summary
Ectopic pregnancy described for the first time in the $11^{\text {th }}$ Century and later on it was described as pregnancy complication. Ectopic pregnancy is potentially life-threatening and remains the leading cause of maternal death. The incidence of ectopic pregnancy is increased during last years all over the world.

Though Laparoscopic surgery is still the cornerstone of treatment in the majority of women, medical management is an alternative treatment option. If the diagnosis of ectopic pregnancy can be made earlier non-invasively, medical treatment with systemic intramuscular Methotrexate (MTX) aimed at reducing mortality, morbidity and reducing costs, minimal intervention/non intervention on comparing with outcomes of surgical treatment. Fertility can be preserved also. Here we presented a case of recurrent ectopic pregnancy with left adnexal mass and stable general condition was treated with MTX successfully.
\end{abstract}

Key words : Medical management, Ectopic pregnancy

\section{Introduction}

An ectopic pregnancy refers to the pregnancy occurring outside the uterine cavity usually in the fallopian tube.

In the past ectopic pregnancies were diagnosed at the time of laparoscopy or surgery, often in women who were unaware that they were pregnant.

Now with the advent of modern diagnostic aids, a pregnancy can easily be diagnosed even before a menstrual period has been missed, as a consequence women can indentified at early gestational age.

Currently over $90 \%$ of ectopic pregnancies can be visualized on Transvaginal scan (TVS). ${ }^{1}$ Early pregnancy units (EPUs) with their access to highresolution TVS and the rapid immunoassay of serum beta human chorionic gonadotrophin ( $\beta$-hCG) allow early diagnosis of pregnancy location.

${ }^{1}$ Dr. Shirin Akhter Assistant Professor Dept. of Obs/Gyn. M.H.Samorita Medical College Hospital

2 Dr. Rumana Nazneen

Associate Professor Dept. of Obs/Gyn

Holy Family Red Crescent

Medical College Hospital

\section{Correspondence}

Dr. Shirin Akhter

Assistant Professor

Dept. of Gynae and Obstetrics

M.H. Samorita Medical College Hospital, Dhaka. spectrum that run from asymptomatic to acute abdomen and hemodynamic shock. The classic clinical triad is: abdominal pain, amenorrhea and vaginal bleeding. Combination of $\beta$-hCG levels and transvaginal ultrasound has a sensitivity of $97 \%$ and specificity of $95 \%{ }^{3}$

Normally, the serum progesterone level in ectopic pregnancy is lower than in normal intrauterine pregnancy. Ultrasound examination represents complex adnexal mass or a solid mass accompanied an ectopic pregnancy, but this could be a corpus luteum, endometrioma, hydrosalpinx, ovarian neoplasia (e.g. dermoid cyst), or a pedunculated myoma. Free liquid in Pouch of Douglas is another sign of ectopic pregnancy, but is not significant of ruptured ectopic. Presence of intra abdominal free fluid shows more often for ruptured ectopic. The exact ultrasound comments are in correlation with $\beta$ hCG levels (discriminatory zone). All viable intrauterine pregnancies can be visualized by transabdominal ultrasonography at a serum $\beta$-hCG levels of higher than $6,500 \mathrm{mIU} / \mathrm{ml}$.

The discriminatory zone for transvaginal ultrasound are reported in levels between 1.000 and 2.000 $\mathrm{mlU} / \mathrm{ml}$.

Missing of intrauterine gestational sac 38 days or more after the last menstrual period or 24 days after conception is the evidence of ectopic pregnancy. ${ }^{4}$

The treatment of ectopic pregnancy includes medical or surgical methods depending on clinical situation, localization of ectopic pregnancy and diagnostic tools.

Medical treatment

The common criteria to include patients in medical treatment with MTX are -

\section{- unruptured pregnancy}

- hemodnamic stability

- adnexal mass $<4 \mathrm{~cm}$

- $\beta$-hCG level less than $5,000 .^{5,6}$

- normal hepatic and renal function

The possibility of ectopic pregnancy rupture is increased, if $\beta$-hCG level is higher after MTX application. ${ }^{7}$ several studies were done with MTX treatment but the success rate depended on the serum level of $\beta$-hCG.

\section{Side effects of MTX}

The mentionable side effects are: alopecia, pelvic pain, nausea, headache weakness, stomatitis, light sensitivity and pneumonia. ${ }^{8}$ Pelvic pain and cramps occurs because of changes happened in ectopic pregnancy other than MTX itself. 


\section{Fertility rate}

There is not any significant difference after surgical or medical treatment of ectopic pregnancy for the future rate of ectopic or intrauterine pregnancy. ${ }^{6,9}$ The fertility rate for intrauterine pregnancy after ectopic pregnancy treated with MTX is $58-61 \%$. The intrauterine pregnancy rate after surgical treatment of ectopic pregnancy is $61-70 \%$ and the recurrence rate of ectopic pregnancy is $18 \%{ }^{2,3}$

\section{Case Report}

Mrs $X$, a 26 years old house wife, from a middle class family, consulted us with the history of 6 wks amenorrhoea with mild lower abdominal pain. Her urine for pregnancy test was +ve. She was further evaluated by USG in OPD which revealed left adnexal mass of about $4.16 \mathrm{~cm} \times$ $2.52 \mathrm{~cm}$ without foetal pole or yolk sac. There was scanty fluid in Pouch of Douglas. Endometrial thickness was $14 \mathrm{~mm}$. her serum- $\beta \mathrm{hCG}$ was $1154 \mathrm{mIU} / \mathrm{ml}$. She had history of right sided salpingectomy due to ruptured tubal pregnancy 2 years back.
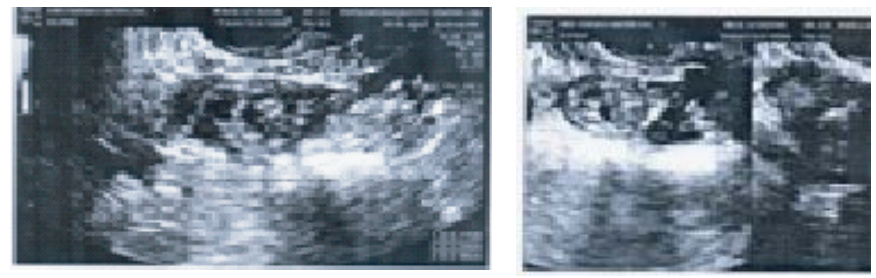

Considering her general condition serum $\beta$-hCG level, two USG reports and previous history, she was selected for medical management with MTX in OPD. Her pre treatment blood biochemical report of CBC, LFT and Renal Function Test was done. $1^{\text {st }}$ dose MTX (1mg/kg of body weight) given and serum $\beta$-hCG level on $D_{4}$ and $D_{7}$ was assessed (1062 $\mathrm{mIU} / \mathrm{ml}$ and $703 \mathrm{mIU} / \mathrm{ml})$. Though level of HCG fall but abdominal pain increased and she was evaluated by another USG which showed increased gestational sac size and pelvic collection.

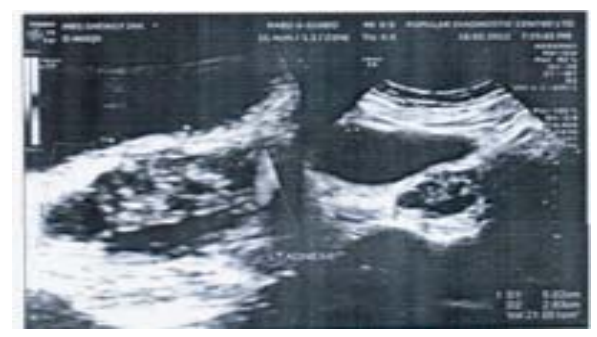

Then $2^{\text {nd }}$ dose of MTX was given and serum $\beta$-hCG level was again assessed on $D_{4 \text { th }}$ and $D_{7 \text { th }}$ level found $469.43 \mathrm{mIU} / \mathrm{ml}$ and 137.11 $\mathrm{mIU} / \mathrm{ml}$ respectively. But unfortunately she developed severe lower abdominal pain, fever and stomatitis. She was hospitalized and treated with antibiotic, analgesics and anxiolytic.

\section{Level of - $\beta$ hCG pre and post treatment of MTX}

InvestigationsPre treatment level

\begin{tabular}{lllllll} 
& & \multicolumn{2}{c}{$1^{\text {st }}$ dose of MTX } & \multicolumn{2}{c}{$2^{\text {nd }}$ dose of MTX } & Post treatment \\
& & D $4^{\text {th }}$ & D $7^{\text {th }}$ & D 4th & D 7th & \\
S $\beta$ hCCG (mIU/ml) & 1154 & 1062 & 703 & 469.43 & 137.11 & $<1$
\end{tabular}

After the clinical condition was improved, she was released from the hospital. Her menstruation returned 2 wks later with average flow. She was advised barrier contraceptives for 6 months. Her serum $\beta$-hCG level became $<1$ after 2 wks of MTX treatment. Resolution of adnexal complex mass occurred about 3 months later. Her recovery period was unevenful.
A follow up Hysterosal pingography was done after 6 months and left tube was found patent. Now she is planning for next pregnancy.

\section{Discussion}

The treatment regime supports the use of Methotrexate as a safe and highly effective alternative treatment of ectopic pregnancies with a success rate of $82 \%$. This highly efficacious result was obtained with strict adherence of the selection criteria. And it allows for only a short hospital stay.

A meta-analysis of non-randomized studies showed success rates of 93\% (95\% CI 89-Methotrexate treatment is very successful for small stable ectopic pregnancies. A meta-analysis of non-randomized studies showed success rates of $93 \%$ (95\% CI 89-96\%) for multi-dose protocols and $88 \%$ (95\% CI $86-90 \%)$ for single dose therapy. Failure of single-dose medical management is associated with initial serum $\beta$-hCG concentrations >5000 IU/l, a moderate or large amount of free fluid on ultrasound, the presence of fetal cardiac activity and a pretreatment increase in serum- $\beta$ hCG of $>50 \%$ over a 48 -hour period. ${ }^{8}$

A retrospective cohort study done between 2003-2008 in "Queen Geraldine" hospital among the admitted patient showed, unruptured ectopic pregnancy was diagnosed in 5.2 week of pregnancy and ruptured ectopic in an average of 6.4 weeks. Surgical intervention was registered in 170 patients and tubectomy was done. Success rate of Methotrexate application was more successful if $\beta$-hCG level was lower. If the $\beta$-hCG level is higher over 10000 the success rate decreased in $83 \%$ and in $\beta$-hCG levels over 15000 the success rate was found to be around $50 \% .^{9}$

Treatment success with MTX was defined as the resolution of the $\beta$-hCG level to less than $5 \mathrm{IU} / \mathrm{L}$. Treatment failure was defined as the need for surgical intervention for any reason.

\section{Conclusion}

The intramuscular Methotrexate is safe and cost effective alternative to the surgical intervention in the unruptured ectopic pregnancies that were diagnosed at early gestation in early pregnancy units and adhere to the strict criteria with no side-effects and the advantage of avoiding invasive surgery. Subsequent tubal patency and reproductive functions were comparable to the surgery. T32herefore, its use is recommend increasingly in the treatment of unruptured ectopic pregnancies.

\section{References}

1. Timor-Tritsch IE. Yeh MN. Peisner DB. Lesser KB. Slavik TA. The use of transvaginal ultrasonography in the diagnosis of ectopic pregnancy. American Journal of Obstetrics \& Gynecology. 161(1):157-61, 1989 Jul.

2. Barnhart $K T$, Sammel MD, Gracia $C R$, et al. Risk factors for ectopic pregnancy in women with symptomatic first trimester pregnancies. Fert Steril. 2006;20:1.

3. Farquhar CM.: Ectopic pregnancy. Lancet. 2005;366:583-91.

4. Kadar N, Bohrer M, Kemmann E, Shelden R.: The discriminatory human chorionic gonadotropin zone for endovaginal sonography: a prospective, randomized study. Fertil Steril. 1994;61:1016.

5. Boyer J, Coste J, Fernandez H, Pouly JL, Job-Spira N.: Si tes of ectopic pregnancy: a 10 year population- based study of 1800 cases. Hum Reprod. 2002; 17:3224.

6. Sowter MC, Farquhar CM, Petrie KJ, Gudex G.: Arandomized trial comparing single dose systemic methotrexate and laparoscopic surgery for the treatment of unruptured ectopic pregnancy. British Journal of Obstetrics \& Gynaecology. 2001;108(2):192-203.

7. Natale A, Candiani M, Barbieri M, Calia C, OdorizziMP OdorizziMP, Busacca M.: Pre-and post-treatment patterns of human chorionic gonadotropin for early detection of persistence after a single dose of methotrexate for ectopic pregnancy. European Journal of Obstetrics and Gynecology and Reproductive Biology. 2004;117:87-92.

8. Schoenfeld A, Mashiach R, Vardy M., Ovadia J. Methotrexate pneumonitis in nonsurgical treatment of ectopic pregnancy. Obstet Gynecol. 1992;80:520-521

9. ernandez $\mathrm{H}$, Gervaise A. Ectopic pregnancy after infertility treatment: modern diagnosis and therapeutic strategy. Hum Report Update 10. 2004:503-513. 